\title{
Digital Technology Adoption in Psychiatric Care: an Overview of the Contemporary Shift from Technology to Opportunity
}

\author{
Ana Hategan ${ }^{1} \cdot$ Caroline Giroux $^{2} \cdot$ James A. Bourgeois ${ }^{3}$
}

Published online: 7 March 2019

(C) Springer Nature Switzerland AG 2019

\section{The Growth of Digital Health: Opportunities to Enhance Clinical Care Delivery}

Technology develops both continuously and discontinuously in an evolutionary process, which quickly moves from one invention to the next through a positive feedback loop of continuous and cumulative improvements. Because the rate of progress of technology is much swifter than often appreciated, it is imperative to think "exponentially" and "expansively" about where we are headed in medicine and how we prepare to integrate emerging technology in clinical and pre-clinical interventions. Technology has opened specific, new frontiers in psychiatry. Mobile devices, like smartphones and tablets, offer clinicians, researchers, and the public alike new ways of providing and receiving clinical treatment, monitoring progress, and increasing understanding of psychiatric illnesses.

The broad categories of "digital health" encompass multiple technological domains, including mobile health (mHealth), health information technology, telehealth (including telemedicine), and wearable medical devices. mHealth includes mobile apps, which are tools that can help individuals promote healthy living, gain access to useful information, and in some circumstances, receive care or manage their own health. mHealth may be part of efforts to improve access, reduce costs, reduce inefficiencies, increase quality of care, and facilitate personalized medicine for patients (U.S. FDA 2018, Digital Health).

In a comprehensive review of telemedicine interventions in primary care, Bashshur et al. (2016) reported on 86 studies

James A. Bourgeois

James.bourgeois@bswhealth.org

1 McMaster University, Division of Geriatric Psychiatry, Hamilton, Ontario, Canada

2 University of California Davis Medical Center, Sacramento, CA, USA

3 Baylor Scott \& White Health, Texas A\&M University Health Science Center, Temple, TX, USA meeting their internal inclusion criteria for analysis. Although there were definitional issues regarding the use of specific telemedicine technologies, overall, most studies supported the use of telemedicine in primary care (Bashshur et al. 2016). Telemedicine methods were often more acceptable to patients than clinicians, were at least as effective as nontechnology enabled interventions, and were increasingly cost-effective (Bashshur et al. 2016).

In recent years, other technological advancements in medicine, particularly the use of mobile technologies and related research, have surged at an unprecedented rate. Sophisticated medical apps have already been developed for smartphones or tablets, and some have been cleared or approved by the U.S. Food and Drug Administration (FDA) (e.g., CardioConfirm, iHealth BG5 Smart Wireless Gluco-Monitoring System) (Food and Drug Administration 2018, Examples of Premarket Submissions). Additionally, HIPPA compliant text messaging apps, such as qliqSOFT (2018), can securely integrate patient EMR information; these apps aim to close the information loop among hospitals, primary care physicians, pharmacies, ancillary staff, and patients.

An important area of interface to master is in the use of technology advances as they link to more "conventional" and "routine" areas of psychiatric care delivery. This can include adding app-derived data to established models of care, such as app-delivered PHQ-9 data to "spreadsheets" in an EMR for management of depressive disorders, including app-enabled medication compliance data into existing pharmacy records, and recording text-messages from patients into the appropriate parts of the EMR to reflect real-time dialog between clinicians and patients (Chan et al. 2014).

As physician-patient texting apps is a new technology, there is not much data yet on physician and patient adoption rates and/or how the application of this technology affects patient outcomes. Some experts in patient engagement believe there can be advantages and drawbacks to physician-patient texting. Although there is no exact substitute for in-person medical visits, there may be the benefit of patient texting specific information to the clinician, e.g., a question about the use 
of an over-the-counter medication. Although we have our smartphones at arm's reach to reply to a text message, there remains the concern that clinicians could become overwhelmed with yet another task they must perform and/ or clinically monitor. There is a need to achieve a balance between being available as clinicians and making things convenient for patients, while ensuring optimal care is not compromised.

There is a growing interest in developing mental health applications (MHapps), and many such apps are already available in iTunes and Android app stores (e.g., PTSD Coach, WhatsUp?). For instance, some MHapps have promised to help the user improve memory function and help the user to connect to a clinician. Other apps might use the device's builtin sensors to collect data on a user's typical behavior patterns, which then may provide a signal that help is needed before a crisis occurs (National Institute of Mental Health 2018).

MHapps can be either active or passive. Active apps require direct participation from the patient, whereas passive apps may access data (e.g., through smartphone functions such as GPS) without the patient or clinician even noticing. Chan et al. (2018) discussed the use of active data from mobile apps in the practice of technology-enabled psychiatry. Those in common use involve "active" data collection, wherein the patient enters relevant data into an app for connection to a clinician. Examples include apps as mood trackers for patient mood self-rating (commonly with app-based prompts and rating scales), diary apps (where patients enter narrative comments on their current state), and wellness apps (where guidance on positive health behaviors that may impact psychiatric illness management are addressed and monitored).

Some app users may not like the potential invasion of privacy associated with data gathering. Patient privacy and security concerns need to be addressed any time technology is used in clinical practice, and (in the USA) it is crucial for this communication to be HIPPA compliant. This applies to apps used in psychotherapy as well. It is also important for clinicians to understand what, if any, data are being collected when a patient uses an app employed in the process of psychotherapy and to ensure patients are informed about this. As in other areas of medical care, in the realm of psychotherapy, clinicians are encouraged to look for apps that incorporate documentation of research on the proposed intervention (e.g., cognitive behavioral therapy), as well as research specifically associated with the app itself.

However, MHapps are not a panacea and clinicians should remain "cautiously enthusiastic" about this new technology. Nevertheless, if we are not familiar with these technologies today as clinicians, it is time to start paying attention because many of our patients are expecting access to them. Because there has been an explosion in the number of mobile apps, particularly MHapps, clinicians do not have time to keep up with all of them. Yet, it is imperative that clinicians take the time to test a specific app themselves before endorsing it to patients and to always ask for feedback from patients on how an app is working for them in order to understand how the use of apps supports broader clinical goals. Before focusing on the state of the information science and the potential to reduce the rates of psychiatric syndromes, it is important to look at some of the advantages and disadvantages of expanding psychiatric treatment and research into mHealth, which could influence the public, patients, and clinicians alike. Table 1 summarizes some advantages and disadvantages of MHapps (National Institute of Mental Health 2018). Addressing potential problems is crucial in ensuring that new MHapps provide benefits while mitigating potential harms.

\section{Future App Development: Including the Patient and Clinical Context}

According to the U.S. FDA, by $2018,50 \%$ of the more than 3.4 billion smartphone and tablet users had downloaded mobile apps (U.S. National Center for Health Statistics 2017). The widespread adoption of mobile technologies certainly is opening innovative ways to improve health research and care delivery. The U.S. National Institute of Mental Health has created the National Advisory Mental Health Council Workgroup on Opportunities and Challenges of Developing Information Technologies on Behavioral and Social Science Clinical Research to track and guide the cutting edge of this rapidly-changing area (National Institute of Health 2018: National Advisory Mental Health Council).

Recent technological developments, including research in suicide prevention, have comprised multiple screening methods, such as network analysis of mobile-phone collected connectivity data, crisis detection from acoustic variability in speech patterns, and automatic detection of suicidality from social media content (Larsen et al. 2015; Mobile Fact Sheet 2018). Digital interventions may be most effective when users are more engaged. Anguera et al. (2016) reported that while mobile technology can be a successful way to recruit research participants, reach a wide section of the population, and is cost-effective, the user's willingness to participate in mobile research protocols (engagement) has remained challenging; study engagement was high during the first 2 weeks of treatment, falling to $44 \%$ adherence by the fourth week. As more studies will use app-based recruitment and treatment as part of their research protocols, finding innovative methods to increase participant retention through increasing motivation and study engagement will be essential to address this important limitation.

MHapps will need to combine the bioengineers' skills for making a user-friendly app which is entertaining with the clinician's skills for providing effective treatment options. It is imperative that patients with the experience of psychiatric 
Table 1 Some facts about advantages and disadvantages of mental health apps (MHapps) (National Institute of Mental Health 2018)

\begin{tabular}{|c|c|}
\hline Advantages & Disadvantages \\
\hline Convenience: & Treatment tailoring: \\
\hline $\begin{array}{l}\text { MHapps may be adopted by those who have difficulty } \\
\text { with in-person clinical appointments or avoided } \\
\text { psychiatric care in the past. Treatment can take place } \\
\text { anytime and anyplace. Individuals can seek treat- } \\
\text { ment options without involving others. }\end{array}$ & $\begin{array}{l}\text { MHapps offer the same treatment program to all users, } \\
\text { but there is need to understand if apps work for all } \\
\text { individuals and for all psychiatric conditions. }\end{array}$ \\
\hline Data collection: & Privacy: \\
\hline $\begin{array}{l}\text { Some apps can gather data without any help from the } \\
\text { user. Receiving information from a large number of } \\
\text { individuals at the same time can increase } \\
\text { researchers' understanding of mental health and } \\
\text { help them develop better interventions. }\end{array}$ & $\begin{array}{l}\text { Apps manage sensitive personal data and app } \\
\text { developers need to be able to guarantee privacy for } \\
\text { app users. }\end{array}$ \\
\hline Affordability: & Regulation: \\
\hline Some apps are cost-free or cost less than usual care. & $\begin{array}{l}\text { There is no industry-wide regulation to inform users if } \\
\text { an app is proven effective; regulation of mental } \\
\text { health technology and the data it generates needs to } \\
\text { be developed. }\end{array}$ \\
\hline Accessibility: & Effectiveness: \\
\hline $\begin{array}{l}\text { Technology can offer treatment to patients in remote } \\
\text { areas or to individuals in times of intense need (e.g., } \\
\text { following a natural disaster). }\end{array}$ & $\begin{array}{l}\text { Scientific evidence that this technology works as well } \\
\text { as the usual care is under-developed. }\end{array}$ \\
\hline Support: & Overrating: \\
\hline $\begin{array}{l}\text { This technology can provide } 24 \text {-h monitoring and/or } \\
\text { intervention support and can complement usual } \\
\text { therapy. }\end{array}$ & $\begin{array}{l}\text { There is concern that if an app promises more than it } \\
\text { delivers, users may turn away from other more } \\
\text { effective therapy programs. }\end{array}$ \\
\hline
\end{tabular}

disorders including a history of suicidal ideation are actively involved in the creation of new apps in order to simulate realworld situations, increase user engagement, and ultimately, increase their effectiveness. We still have to learn from those who survived suicide attempts (e.g., what pushed them "over the edge," what helped them, what makes them glad they have survived, what constituted resilience).

It remains unknown whether the currently developed MHapps are evidence-based and whether they contain potentially harmful content. A recent systematic review of 123 apps on both the Android and iOS platforms that screened and reviewed their app content has shown that some did provide elements of best practice, but none provided comprehensive evidence-based support (Larsen et al. 2016). The abundance of apps on the market has driven initiatives, such as the Mobile Applications Rating Scale (MARS) (Stoyanov et al. 2015). The MARS is a simple, objective, and reliable tool for classifying and assessing the quality of mobile health apps, with an excellent internal consistency (alpha $=0.90)$ and interrater reliability intraclass correlation coefficient $(\mathrm{ICC}=$ 0.79) (Stoyanov et al. 2015). When conducting systematic reviews, time-dependent nature of terminology use in mHealth research must consider terminology change. For example, in earlier to more recent years, terminology changed from "mobile phone" to "smartphone" and from "applications" to "apps." Furthermore, apps with potentially harmful content were also identified (Larsen et al. 2016).
Pending further research, clinicians should be cautious in recommending specific apps, because potentially harmful content can be presented as helpful. The American Psychiatric Association (APA) has provided a free mobile apps evaluation tool, although not explicitly a rating app, to help psychiatrists select and rate an app, which they may choose to integrate in the care of their patients; this is based on the dictum of "do no harm" as well as a risk-benefit analysis (American Psychiatric Association). Beyond gathering basic background information on the mobile app by the clinician, the main areas covered in this APA evaluation model are: (1) safety/privacy, (2) evidence (e.g., effectiveness), (3) ease of use, and (4) interoperability (American Psychiatric Association 2018).

The FDA encourages the development of software-based technologies, including mobile apps (also termed by FDA and other regulators as "software as a medical device," or SaMD) (U.S. FDA 2018, Digital Health). Nonetheless, the FDA has a public health responsibility to oversee the safety and effectiveness of medical devices. After careful consideration, the FDA has released a series of policy guidance that explains the agency's oversight of specific mobile medical apps as meeting criteria for regulated medical devices, since not all medical apps require government regulation (U.S. FDA 2018, Digital Health). The FDA has issued others important policies including aiming to create a more streamlined precertification process coupled with a better leveraging post-marketing data collection on the device's safety and effectiveness (U.S. FDA 
2018, Digital Health). Moreover, the FDA has helped establish basic policies for developing an internationally harmonized regulatory framework for SaMD that endeavors to better meet the needs of the rapid innovation cycles and business models of software developers in the medical field (U.S. FDA 2018, Digital Health).

\section{Data-Driven Science in Psychiatry and the Use of MHApps}

There have been various studies on the efficacy of MHApps in enabling the treatment of psychiatric illness, most commonly depressive and anxiety disorders. While methodologies vary, these studies have been subject to meta-analysis, which leads to optimism regarding the integration of MHApps into the treatment of these important and common psychiatric illnesses. Firth et al. (2017a) conducted meta-analysis of 18 eligible randomized controlled trials (RCTs) of 22 smartphone apps, with over 3000 total participant patients with depressive symptoms. Patients using smartphone apps showed a greater improvement in symptoms vs. controls, with a greater effect when compared with inactive than active control conditions. Studies of cognitive training apps (which may have positive impact on depressive symptoms) had a significantly smaller effect size on depressive symptoms than those apps focusing on mental health per se. Mood monitoring methodologies, interventions founded in cognitive behavioral therapy, and mindfulness apps did not have significant effects.

In a similarly designed meta-analysis of RCTs using smartphone apps in the management of anxiety symptoms and/or anxiety disorders, Firth et al. (2017b) reported on nine eligible RCTs, with over 1800 total participants. Statistically significantly greater reductions in total anxiety scores were seen with the groups with smartphone interventions vs. control conditions $(g=0.325,95 \% \mathrm{CI}=0.17-0.48, p<0.01)$, with no evidence of publication bias. Effect sizes from smartphone interventions were significantly greater compared with waitlist/inactive controls than active control conditions.

With the rise in disclosure of sexual trauma by survivors and the reactivating effect of past trauma in the public who hears about those stories, we have to be prepared in our clinical settings to provide support and offer treatment modalities that will meet the increased needs of individuals suffering from trauma-related disorders. Additionally, post-traumatic stress disorder (PTSD) is associated with suicidal ideation and suicide attempts. Because of their versatility, technologies can enable mental health professionals to serve greater numbers of patients by expanding and improving access to treatment modalities to address distress and suicide risk.

Home-based telemental health (HBTMH) is empowering because it can allow trauma survivors to receive mental health services in a manner and location based on their preference.
That alone is a trauma-informed approach and meets the standard of care. Additionally, HBTMH might initially decrease the exposure to triggers and would lay the foundation of trust necessary for expanding one's environment when coping with avoidance behaviors as the next therapeutic goal during graduated exposure. For instance, VA medical centers are maledominated settings with potentially difficult trauma cues for female veterans who have experienced military sexual trauma (Azarang et al. 2018).

Azarang et al. (2018) listed various MHapps and their applicability in individuals who have experienced trauma. The PTSD Coach app is a helpful psychoeducational and selfmanagement tool for trauma survivors, which normalizes distress, helps individuals cope with acute symptoms, provides information about PTSD and treatment options, and encourages self-assessment. The Prolonged Exposure (PE) Coach is an app that can be used concurrently with psychotherapy. It allows users to audio-record therapy sessions and track PTSD symptoms over time. The dialectical behavior therapy coach app is used to treat individuals with borderline personality disorder and substance-related disorders.

There is a current need to rethink traditional mental health service structures and bioengineering capacity to foster the use of safe, secure, and robust digital apps within mental healthcare systems, while encouraging ongoing research to meet the needs of sustainable medicine. Ascertainment of app effectiveness remains a challenge. This new technological industry presents an uncertain frontier for users and healthcare professionals. While the apps become more appealing and user-friendly, there still is not a lot of data on their effectiveness. Most apps do not have peer-reviewed research to support their clinical claims, and it is unlikely that every MHapp will go through a rigorous scientific trial to test effectiveness. One reason is that testing can be a time-consuming process, while technology evolves quickly and becomes outdated fast.

\section{Suicide Risk Management: a Specific Area for MHApps}

Suicide should be viewed as not only a "psychiatric problem," but rather one of public health more globally. While clearly important in the phenomenology of psychiatric illness (particularly depressive disorders, bipolar disorders, psychotic disorders, neurocognitive disorders, and certain personality disorders), suicide risk is also increased in other CNS or systemic medical illnesses (e.g., multiple sclerosis, Huntington disease, seizure disorder, renal failure, HIV disease). As such, there is an imperative to provide psychiatry input as the leaders, but not the sole owners of the suicide prevention strategies. Any progress in suicide assessment that may derive from the innovations likely to come from departments of psychiatry should 
be actively shared with other medical departments (e.g., neurology, internal medicine, endocrinology).

This article brings attention to the need to skillfully develop and integrate evolving mobile technology into the pre-clinical and clinical "real world" of suicide prevention, to conduct active research into the evidence base of this technology, and to advocate for "sustainable medicine" in which physicians learn to integrate new technologies into their medical practice. Therefore, apps may be of supplemental use in suicide prevention and suicide risk management.

Recent research from the Centers for Disease Control and Prevention (CDC) has shown that since 1999 the suicide rate in the USA grew by more than $25 \%$, with suicide being the 10th leading cause of death in 2015 (mHealth Economics 2017). Some suicides can be preventable with timely preclinical and clinical intervention; however, somewhere in the world, a person dies by suicide every $40 \mathrm{~s}$, and many more people attempt suicide (National Institute of Mental Health 2018). Suicidal behavior occurs throughout the lifespan and is pervasive across the world. However, among young people aged 15-29, suicide is the second leading cause of death globally; this is a demographic that is juxtaposed with the highest segment of the U.S. population to own smartphones and to use mobile apps (mHealth Economics 2017; National Institute of Health 2018). This convergence of demographic and social trends thus leads to opportunities to apply technology to the vexing problem of suicide. The increasing effort for understanding and predicting suicide using new digital technologies could make a great deal of sense when trying to reach patients who are vulnerable to suicide, by use of technology already embraced by this population.

Fleischmann et al. (2008) reported on an international study of a brief suicide prevention intervention deployed in emergency departments treating suicide attempters, where a RCT compared a brief intervention and contact (BIC) specifically to mitigate suicide risk (which included patient education and follow up) to treatment as usual (TAU). They found a rate of completed suicide at 18 month follow-up of $0.2 \%$ in the intervention group vs. TAU, significant at the $p<0.001$ level. App-based suicide mitigation interventions may benefit from a design similar to this. A subsequent meta-analysis of RCTs for suicide prevention by Riblet et al. (2017) found three RCTs of the WHO BIC model to significantly lower the risk of suicide, while similar studies of CBT and lithium did not have a significant effect on risk of completed suicide.

Quantitative data-driven science has the potential to provide clinicians with useful digital tools to dynamically assess suicide risk and potentially to mitigate this risk. Many risk factors for suicide are known, and clinicians have to rely on clinical acumen to assess suicide risk. However, suicide risk assessment in real-world clinical practice involves a significant degree of subjectivity. Developing an objective model to predict suicide, although highly desirable, has proven to be difficult for various reasons. Suicide risk is dynamic, based on modifiable combinations of a multitude of biological, psychological, and social factors. Some individuals may be chronically at high risk of suicide, but an acute life event could rapidly escalate that risk further. Thus, someone who is at relatively low risk could rapidly become at imminent risk of suicide due to an acute life stressor.

By collecting and analyzing large population data and patient data, digital technology could theoretically help clinicians to identify and quantify useful objective predictors in clinical suicide risk assessment. This includes information on: (1) the singular impact of each suicide risk factor versus combined interaction of various suicide risk factors; (2) the individualized suicide risk profile of a given person; and (3) the immediate risk of a person at any given time with consideration of newly acquired information regarding nuances of suicidality (Vahabzadeh et al. 2016). In this view, efforts to increase objectivity in suicide risk assessment have been launched by increasing research interest in adopting the digital technology. Two recent studies have aimed to shed light in the area of suicide prediction in both male and female psychiatric patient populations that combined data from mood-focused smartphone apps (Convergent Functional Information for Suicidality (CFI-S) and Simplified Affective State Scale (SASS)) with genomic data (blood gene expression biomarkers) (Levey et al. 2016; Niculescu et al. 2015). By combining the results of both approaches, researchers were able to identify some useful objective prediction tests for suicidal ideation and for future hospitalizations for suicidality. However, there were several study limitations, including the need to further test how such predictors apply to the general population and in different clinical settings. In these two studies, the clinical information apps CFI-S and SASS have shown good predictive ability for suicidal ideation and future hospitalizations for suicidality in bipolar disorder, major depressive disorder, schizoaffective disorder, and schizophrenia, with an area under the curve (AUC) of $89 \%$ for CFI-S and AUC of $85 \%$ for SASS, respectively (Levey et al. 2016; Niculescu et al. 2015). Such combined technologies could potentially help clinicians conduct better risk assessment, tracking, and prediction of suicidality (ideation and behavior). Studies with larger numbers and longer follow-up are necessary.

In addition, several mHealth apps have been developed with the sole objective to empower primary care and mental health clinicians to provide effective, evidence-based care for a variety of psychiatric conditions. For example, SAMHSA (Substance Abuse and Mental Health Services Administration) has created free mHealth apps with the goal to offer access to treatment and prevention tools for several topics including suicide; their Suicide Safe app is a learning tool based on the SAMHSA's Suicide Assessment Five-Step Evaluation and Triage (SAFET) approach (U.S. Department of Health and Human Services 2019). 


\section{Digital Suicide Prevention: the Next Steps}

A paradigm shift in medicine leads the way for both healthcare providers and patients to adapt to the current technological advances as key ingredients. Aside the call for further research into the evidence/effectiveness of mobile apps, the following objectives may play important roles in the development of software products for mental health, including global suicide prevention efforts, which aim to:

1. Work on any device in order to generalize world-wide access (e.g., smartphone, tablet, or internet platforms);

2. Allow incorporation of remote psychotherapy (e.g., through smartphone or online platform) and face-to-face contact in order to provide a balance between technology and the "human touch" (i.e., active vs. passive assessment/monitoring);

3. Promote engagement of users when delivering therapies or skill development (e.g., through game-like approaches);

4. Encourage the application of real-time apps (e.g., users exchanging information with peers/professionals)

Other future speculative areas of potential development regarding MHapps lead to several questions, including:

1. Can apps be connected to an alert system (with a prompt notification of the physician or other clinician) when acute suicide risk suddenly increases?

2. Can MHapp-derived patient data be integrated into an algorithm leading patients to crisis management services and other urgent-care resources?

3. Can MHapps be connected to feedback methods such as biofeedback? For instance, when there are suicidal thoughts, can there be a reminder to meditate, exercise, call a friend, and/or monitor the physiological state?

4. Can MHapps include other specific behavioral advice, such as reminding patients to do a gratitude list or use an emergency self-care "tool kit"?

5. Can MHapps be programmed to lead the patient to an exercise in cognitive restructuring, such as asking "What went well today?" to shift perspective for the acute crisis patient?

6. For geriatric psychiatry, are there gero-friendly technologies for older populations who are at higher risk of suicide but who are not tech-savvy?

7. Can patients give consent for their suicide risk data to be transmitted to a trustful contact who would intervene or check on them as needed?

Addressing and preemptively anticipating such concerns will be important as psychiatry leads the way in general adaptation of MHapps to enhance the practice of suicide risk assessment and management. Close partnerships with app developers and psychiatric researchers will be essential as this exciting and transformative InfoTech-enabled period in clinical services history develops and flourishes.

\section{Integration of Technology Adoption into Psychiatry Education}

It is important for academic programs (e.g., M.D./D.O. programs, other graduate level professional training programs, graduate medical education programs) to actively integrate mastery of technological advances such as wearable medical devices and mobile apps into training programs. It is important for academic leaders and regulatory bodies to consider mastery of clinically related technology advances as intrinsic to learning how to deliver clinical care. Gone are those days where a psychiatry department could deploy one or two faculty members as "the telemedicine service"; in the current environment, these areas must be considered an area of mastery for most (if not all) faculty members and trainees. Therefore, integration of the use of clinical-care enhancing technology becomes a pedagogic, as well as patient care, imperative.

Hilty et al. (2015) reviewed the adoption of basic core competencies in telepsychiatry and other technology-assisted clinical methodologies. Since telepsychiatry and other technology-assisted clinical methods overlap, this group recommended that competency expectations integrate telemedicine into other technology advancements and that the level of technology use sophistication (from novice to expert levels) be integrated into the competency determinations of clinicians.

A trainee-specific pedagogical imperative is needed to promote integration of the use of clinical-care enhancing technology in the care of patients. This should extend beyond the boundaries of training in psychiatry as a medical specialty in order to seamlessly interface with allied health professional development. Furthermore, we propose the conception and implementation of an "assistant program director for technology adoption" role for psychiatry residency training programs to increase awareness and adoption of new technologies into clinical practice. We also encourage departments of psychiatry to partner with departments of computer science and other technical fields to foster collaborative academic opportunities.

Similarly, the embrace of technology needs to be thought of as a "broad-based continuum," not just limited to video-based visits or app supported data gathering. Analogous perhaps to "neuromodulation centers" of clinical psychiatry (where ECT, rTMS, and ketamine are used), the "technology center" of a psychiatry department should feature video-based visits, use of clinical simulators, application of virtual reality devices, and mobile apps, electronic/virtual consults, advanced application of EMT technology, as well as yet-to-emerge 
technology. The academic opportunities for departments of psychiatry to partner with departments of computer science and other technical fields may someday prove as valuable as more classic collaborative relationships with departments of neurology, neurosurgery, pharmacy, and neurosciences.

Compliance with Ethical Standards Yes re authorship, N/A re research.

\section{Ethical Considerations N/A}

Disclosure of Funding Sources None.

Publisher's Note Springer Nature remains neutral with regard to jurisdictional claims in published maps and institutional affiliations.

\section{References}

American Psychiatric Association. (2018). App evaluation model. https:// www.psychiatry.org/psychiatrists/practice/mental-health-apps/appevaluation-model. Accessed 30 Sept 2018.

Anguera, J. A., Jordan, J. T., Castaneda, D., Gazzaley, A., \& Areán, P. A. (2016). Conducting a fully mobile and randomised clinical trial for depression: Access, engagement and expense. BMJ Innovations, 2(1), 14-21.

Azarang, A., Pakyurek, M., Giroux, C., Nordahl, T., \& Yellowlees, P. (2018). Information technologies: An augmentation to post-traumatic stress disorder treatment among trauma survivors. Telemedicine Journal and EHealth. https://doi.org/10.1089/tmj.2018.0068.

Bashshur, R. L., Howell, J. D., Krupinski, E. A., Harms, K. M., Bashshur, N. B., \& Doarn, C. R. (2016). The empirical foundations of telemedicine interventions in primary care. Telemedicine Journal and E-Health, 22(5), 342-375.

Chan, S. R., Torous, J., Hinton, L., \& Yellowlees, P. (2014). Mobile telemental health: Increasing applications and a move to hybrid models of care. Healthcare (Basel), 2(2), 220-233.

Chan, S. R., Burke Parish, M., Fazio, S., \& Torous, J. (2018). Data collection from novel sources. In P. Yellowlees \& J. M. Shore (Eds.), Telepsychiatry and health technologies: A guide for mental health professionals (pp. 183-226). Arlington VA: American Psychiatric Association Publishing.

Firth, J., Torous, J., Nicholas, J., Carney, R., Pratap, A., Rosenbaum, S., \& Sarris, J. (2017a). The efficacy of smartphone-based mental health interventions for depressive symptoms: A meta-analysis of randomized controlled trials. World Psychiatry, 16(3), 287-298.

Firth, J., Torous, J., Nicholas, J., Carney, R., Rosenbaum, S., \& Sarris, J. (2017b). Can smartphone mental health interventions reduce symptoms of anxiety? A meta-analysis of randomized controlled trials. Journal of Affective Disorders, 218, 15-22.

Fleichmann, A., Bertolote, J. M., Wasserman, D., De Leo, D., Bolhari, J., Botega, N. J., et al. (2008). Effectiveness of brief intervention and contact for suicide attempters: A randomized controlled trial in five countries. Bulletin of the World Health Organization, 86(9), 703-709.
Food and Drug Administration (FDA). (2018). Examples of pre-market submissions that include MMAs cleared or approved by FDA. FDA website. https://www.fda.gov/MedicalDevices/DigitalHealth/ MobileMedicalApplications/ucm368784.htm. Accessed 30 Sept 2018.

Hilty, D. M., Crawford, A., Teshima, J., Chan, S., Sunderji, N., Yellowlees, P. M., Kramer, G., O'neill, P., Fore, C., Luo, J., \& Li, S. T. (2015). A framework for telepsychiatric training and e-health: Competency-based education, evaluation, and implications. International Review of Psychiatry, 27(6), 569-592.

Larsen, M. E., Cummins, N., Boonstra, T. W., O’Dea, B., Tighe, J., Nicholas, J., et al. (2015). The use of technology in suicide prevention. Conference Proceedings: Annual International Conference of the IEEE Engineering in Medicine and Biology Society, 2015, , 7316-7319.

Larsen, M. E., Nicholas, J., \& Christensen, H. (2016). A systematic assessment of smartphone tools for suicide prevention. PLoS One, 11(4), e0152285.

Levey, D. F., Niculescu, E. M., Le-Niculescu, H., Dainton, H. L., Phalen, P. L., Ladd, T. B., et al. (2016). Towards understanding and predicting suicidality in women: Biomarkers and clinical risk assessment. Molecular Psychiatry, 21(6), 768-785.

mHealth Economics. (2017). Current status and future trends in mobile health. https://research2guidance.com/product/mhealth-economics2017-current-status-and-future-trends-in-mobile-health/. Accessed 30 Sept 2018.

Mobile fact sheet. (2018). http://www.pewinternet.org/fact-sheet/mobile/. Accessed 30 Sept 2018.

National Center for Health Statistics. (2017). Health, United States, 2016: With chartbook on long-term trends in health. Hyattsville, MD. https://www.cdc.gov/nchs/data/hus/hus16.pdf\#019. Accessed 30 Sept 2018.

National Institute of Health. (2018). National Advisory Mental Health Council (NAMHC) workgroups. https://www.nimh.nih.gov/about/ advisory-boards-and-groups/namhe/namhc-workgroups/index. shtml. Accessed 30 Sept 2018.

National Institute of Mental Health. (2018). Technology and the future of mental health treatment. https://www.nimh.nih.gov/health/topics/ technology-and-the-future-of-mental-health-treatment/index.shtml. Accessed 30 Sept 2018.

Niculescu, A. B., Levey, D. F., Phalen, P. L., Le-Niculescu, H., Dainton, H. D., Jain, N., et al. (2015). Understanding and predicting suicidality using a combined genomic and clinical risk assessment approach. Molecular Psychiatry, 20(11), 1266-1285.

qliqSOFT. (2018). https://www.qliqsoft.com/. Accessed 30 Sept 2018.

Riblet, N. B. V., Shiner, B., Young-Xu, Y., \& Watts, B. V. (2017). Strategies to prevent death by suicide: Meta-analysis of randomised controlled trials. British Journal of Psychiatry, 210(6), 396-402.

Stoyanov, S. R., Hides, L., Kavanagh, D. J., Zelenko, O., Tjondronegoro, D., \& Mani, M. (2015). Mobile app rating scale: A new tool for assessing the quality of health mobile apps. JMIR mHealth and uHealth, 3(1), e27.

U.S. Department of Health and Human Services. (2019). Substance Abuse and Mental Health Services Administration (SAMHSA). https://store.samhsa.gov/apps/suicide-safe. Accessed 12 Feb 2019.

U.S. FDA. (2018). Digital health. https://www.fda.gov/medicaldevices/ digitalhealth/. Last updated July 27, 2018. Accessed 30 Sept 2018.

Vahabzadeh, A., Sahin, N., \& Kalali, A. (2016). Digital suicide prevention: Can technology become a game-changer? Innovations in Clinical Neuroscience, 13(5-6), 16-20. 